\title{
NOUVELLE
}

\section{Conversions épigénétiques transmises de façon stable au cours des générations}

Augustin de Vanssay, Anne-Laure Bougé, Antoine Boivin, Catherine Hermant, Laure Teysset, Valérie Delmarre, Christophe Antoniewski, Stéphane Ronsseray
Laboratoire biologie du développement (LBD), UMR7622, CNRS/université Pierre et Marie Curie, 9, quai Saint-Bernard, 75252 Paris Cedex 05, France. stephane.ronsseray@snv.jussieu.fr christophe.antoniewski@snv.jussieu.fr

Ces petits ARN permettraient une « communication », dont le mécanisme est à ce jour inconnu, entre les épiallèles $B^{\prime}$ et $B-I$ présents au sein d'un même génome. La paramutation peut ainsi provoquer une propagation très rapide, au sein d'une population, d'un nouvel épiallèle ayant des propriétés paramutagéniques. عlle a été décrite chez plusieurs espèces végétales (maïs, petit pois, tomate, etc.). Des transmissions par conversion épigénétique ont été également décrites chez la souris [4]. Cependant, ces dernières sont beaucoup moins stables: elles ne se transmettent pas au-delà de quatre générations.

Répression des éléments transposables par les piARN dans la lignée germinale chez l'animal

Les éléments transposables sont des séquences d'ADN capables de se déplacer d'un site chromosomique à un autre. Ils sont le plus souvent présents sous forme de nombreuses copies dispersées sur l'ensemble du génome. Ces éléments étant générateurs de mutations et de cassures chromosomiques, leur mobilité doit être régulée pour préserver l'intégrité du génome. L'étude de la régulation d'un élément transposable a récemment permis de mettre en évidence un cas de paramutation chez la drosophile (Drosophila melanogaster) [5]. Cette paramutation est très stable au cours des générations et peut être induite de façon récurrente. Elle est liée à la transmission maternelle de petits ARN non codants et s'accompagne d'un « apprentissage » qui 


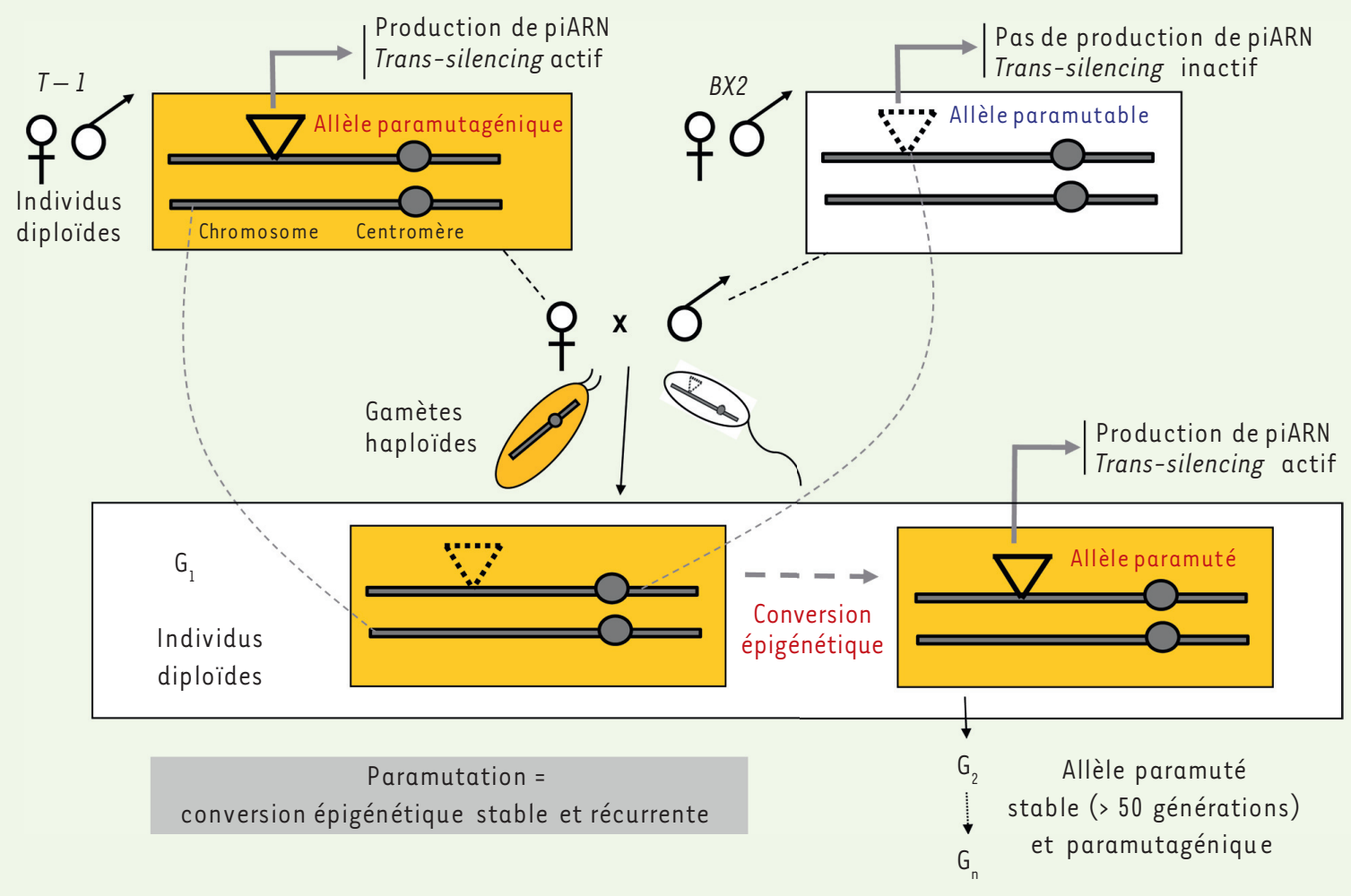

Figure 1. Paramutation chez la drosophile. Deux types de lignées de drosophiles portant les mêmes répétitions de transgènes en tandem (cluster) présentent des propriétés épigénétiques différentes. Dans une première lignée $(T-1)$, le cluster (allèle paramutagénique, triangle plein) produit des piARN ovariens (24-29 nt). Dans une seconde lignée (BX2), le cluster (allèle paramutable, triangle pointillé) n'en produit pas ou très peu. Le croisement de femelles $T-1 /+$ avec des mâles $B X 2 /+$ peut produire des femelles $G_{1}$ ayant reçu de leur mère un cytoplasme contenant des piARN (fond orange), mais ne possédant pas l'allèle paramutagénique, et ayant reçu de leur père l'allèle paramutable. Dans ces femelles $G_{1}$, l'allèle paternel est paramuté : il se met à produire des piARN et peut exercer un trans-silencing de séquences homologues au sein du génome. L'allèle ainsi paramuté est transmis de façon stable au cours des générations, et est capable à son tour de convertir un allèle paramutable.

confère au locus paramuté la capacité de produire à son tour ces mêmes petits ARN dans les ovaires. Ces petits ARN seront ainsi transmis à la génération suivante. Ce phénomène correspond au premier cas stable de paramutation décrit dans le monde animal.

Les populations naturelles de la mouche du vinaigre ont été envahies au XXe siècle par un élément transposable appelé élément $P$. Cet élément mobile, présentant une forte capacité à transposer dans la lignée germinale et ainsi à se multiplier au sein des génomes, s'est propagé en moins de 20 ans au sein de toutes les populations naturelles de drosophiles dans le monde $[6,7]$. Cette diffusion, qui implique des phénomènes migratoires, a vraisemblablement été facilitée par les activités humaines. Après une phase intense de transposition, provoquant mutations et cassures chromosomiques, une répression de la transposition de l'élément $P$ s'est mise en place dans les populations de drosophiles [8]. Nous nous sommes intéressés à la mise en place de cette répression et avons montré qu'une seule copie de $P$, insérée dans la chromatine compactée à proximité des télomères (hétérochromatine télomérique), est capable de réprimer plusieurs dizaines de copies actives de l'élément $P$ présentes dans le même génome [9, 10]. Cette répression en trans est liée à la présence, dans les ovaires, de petits ARN non codants de 24 à 29 nucléotides, appelés piARN (PIWI-interacting RNA), qui sont transmis par le cytoplasme de l'ovocyte [11]. Cette voie de répression par les piARN a été découverte chez la drosophile
[12]. Elle régule non seulement l'activité des éléments transposables dans la lignée germinale, mais aussi l'expression de certains gènes au cours du développement [13]. Son existence est très ancienne et elle est conservée chez les vertébrés [14].

Un système de transgènes a été utilisé par la suite pour étudier les propriétés phénotypiques, génétiques et moléculaires de la répression de l'élément $P$. Dans ce système, appelé trans-silencing, un transgène inséré dans l'hétérochromatine télomérique réprime, dans la lignée germinale, un transgène homologue, quelle que soit la localisation chromosomique de ce dernier [15, 16]. Le trans-silencing fonctionne même si l'homologie entre l'élément télomérique (le répresseur) et l'élément réprimé (la cible) concerne une 
séquence différente de l'élément $P$ (par exemple le gène lacZ de $\varepsilon$. coli) [17]. Ceci suggère que ce système de répression génique est très général.

\section{Paramutation stable liée}

à l'émergence d'un locus producteur de piARN chez la drosophile

Nous avons également montré que d'autres locus génomiques hétérochromatiques sont capables de réaliser un trans-silencing de ce type dans la lignée germinale. Ainsi, un locus composé de transgènes répétés en tandem (cluster) s'est révélé particulièrement intéressant. $\varepsilon$ n effet, nous disposons de deux lignées possédant sept copies d'un transgène insérées en tandem en un même locus chromosomique : la lignée $T$ - 1 , capable d'induire le trans-silencing, et la lignée $B X 2$, incapable d'induire cette répression [18]. Les clusters de transgènes de $T-1$ et $B X 2$ sont génétiquement identiques (I'un dérive de l'autre) [19]. T-1 produit des piARN dans les ovaires, que nous avons caractérisés par séquençage à haut débit, tandis que $B X 2$ n'en produit qu'une quantité infime. Ce locus transgénique présente ainsi, selon la lignée qui l’héberge, des propriétés épigénétiques différentes qui sont transmises de façon totalement stable au cours des générations.

De façon surprenante, une drosophile femelle $B X 2 /+\left(G_{1}\right)$ ayant reçu de sa mère $T-1 /+$ le cytoplasme contenant des piARN, mais pas le cluster $T-1$ (qui produit les piARN), et ayant reçu de son père $B X 2 /+$ le cluster $B X 2$ (qui ne produit pas de piARN), présente une capacité de trans-silencing totale, similaire à celle de sa mère (Figure 1 ). De plus, une femelle $B X 2 /+\left(G_{2}\right)$ issue de cette femelle $B X 2 /+\left(G_{1}\right)$ présente également une capacité de trans-silencing totale, et synthétise autant de piARN ovariens qu'une femelle $T-1 /+$. Ainsi, mis au contact d'un cytoplasme contenant des piARN, un cluster $B X 2$ devient lui-même un fort producteur de piARN et peut à son tour réaliser un trans-silencing total, et ce de façon stable au cours des générations (ceci a été testé durant 55 générations). De plus, ces femelles épigénétiquement « converties », appelées $B X 2 * /+$, produisent des ovocytes dont le cytoplasme est à son tour capable d'enclencher la production de piARN par un cluster $B X 2$ inerte, lors de croisements avec des mâles $B X 2 /+$. Ce dernier cluster inerte devient lui-même un producteur stable de piARN répresseurs: l'élève $B X 2 *$ est donc devenu professeur. Ces piARN ont été caractérisés d'un point de vue moléculaire par séquençage à haut débit et les capacités répressives d'un locus $B X 2{ }^{*}$ sont abolies par une mutation affectant aubergine, l'un des acteurs principaux de la voie des piARN, ce qui confirme l'implication de cette voie dans cette répression. Enfin, un locus $B X 2$ inerte peut également être activé par un locus producteur de piARN localisé sur un chromosome différent, ce qui généralise l'impact possible de ce type d'interaction au sein du génome.

Ce travail met en évidence pour la première fois un authentique mécanisme de paramutation dans le règne animal. Il est important de souligner que cette paramutation s'opère par simple transfert d'informations présentes dans le cytoplasme de l'ovocyte, c'est-à-dire qu'elle se réalise sans que le chromosome maternel paramutagénique ne soit jamais mis en présence du chromosome paramuté dans les cellules des descendants. Ainsi, une authentique paramutation s'avère réalisable en absence de toute possibilité d'appariement entre les locus paramutagénique et paramuté, ce qui était inédit, y compris dans le monde végétal.

L'étude de la paramutation est fondamentale, d'une part pour la connaissance des supports moléculaires impliqués dans I'hérédité non génétique et, d'autre part, pour l'analyse du mode d'émergence des locus producteurs de piARN. Notre travail ouvre également de nouvelles pistes d'investigation des mécanismes de propagation des nouveautés épigénétiques au sein des populations. $\diamond$

Inheritance of paramutation in plants and Drosophila

\section{LIENS D'INTÉRÊT}

Les auteurs déclarent n'avoir aucun lien d'intérêt concernant les données publiées dans cet article.

\section{RÉFÉRENCES}

1. Brink RA. A genetic change associated with the $R$ locus in maize which is directed and potentially reversible. Genetics $1956 ; 41: 872-89$.

2. Coe $\varepsilon H$. A regular and continuing conversion-type phenomenon at the B locus in maize. Proc Natl Acad Sci USA $1959 ; 45$ : 828-32.

3. Chandler VL. Paramutation: from maize to mice. Cell $2007 ; 128: 6415$.

4. Rassoulzadegan M, Grandjean V, Gounon P, et al. RNAmediated non-Mendelian inheritance of an epigenetic change in the mouse. Nature 2006 ; $441: 469-74$.

5. de Vanssay A, Bougé AL, Boivin A, et al. Paramutation in Drosophila linked to emergence of a piRNAproducing locus. Nature $2012 ; 490: 112-5$.

6. Kidwel MG, Kidwell JF, Sved JA. Hybrid dysgenesis in Drosophila melanogaster: a syndrome of aberrant traits including mutation, sterility, and male recombination. Genetics $1977 ; 86: 813-33$.

7. Anxolabehere D, Kidwell MG, Periquet G. Molecular characteristics of diverse populations are consistent with the hypothesis of a recent invasion of Drosophila melanogaster by mobile P elements. Mol Biol Evol 1988 ; $5: 252-69$.

8. Engels WR. Hybrid dysgenesis in Drosophila melanogaster: rules of inheritance of female sterility. Genet Res 1979; $33: 219-36$.

9. Marin L, Lehmann M, Nouaud D, et al. P-Element repression in Drosophila melanogaster by a naturally occurring defective telomeric P copy. Genetics $2000 ; 155$ : 1841-54.

10. Ronsseray $S$, Lehmann $M$, Nouaud $D$, Anxolabéhère $D$. The regulatory properties of autonomous subtelomeric $P$ elements are sensitive to a suppressor of variegation in Drosophila melanogaster. Genetics $1996 ; 143: 1663-74$.

11. Brennecke J, Malone CD, Aravin AA, et al. An epigenetic role for maternally inherited piRNAs in transposon silencing. Science 2008 ; 322 : 1387-92.

12. Brennecke J, Aravin AA, Stark A, et al. Discrete small RNA-generating loci as master regulators of transposon activity in Drosophila. Cell 2007 ; 128 : 1089-103.

13. Rouget C, Papin C, Boureux A, et al. Maternal mRNA deadenylation and decay by the piRNA pathway in the early Drosophila embryo. Nature $2010 ; 467$ : 1128-32.

14. Zamudio N, Bourc'his D. Transposable elements in the mammalian germline: a comfortable niche or a deadly trap? Heredity (Edinb) $2010 ; 105: 92-104$.

15. Josse T, Maurel-Zaffran C, de Vanssay A, et al. Telomeric trans-silencing in Drosophila melanogaster: tissue specificity, development and functional interactions between non-homologous telomeres. PLoS One 2008 ; 3 : e3249.

16. Josse T, Teysset L, Todeschini AL, et al. Telomeric trans-silencing: an epigenetic repression combining RNA silencing and heterochromatin formation. PLOS Genet 2007 ; 3 : 1633-43.

17. Pöyhönen M, de Vanssay A, Delmarre V, et al. Homologydependent silencing by an exogenous sequence in the Drosophila germline. G3 (Bethesda) $2012 ; 2$ : 331-8.

18. Ronsseray S, Boivin A, Anxolabehere D. P-Element repression in Drosophila melanogaster by variegating clusters of P-lacZ-white transgenes. Genetics 2001; 159 : 1631-42.

19. Dorer DR, Henikoff S. Transgene repeat arrays interact with distant heterochromatin and cause silencing in cis and trans. Genetics $1997 ; 147: 1181-90$. 